\title{
Every-Other-Day Valganciclovir Prophylaxis for Cytomegalovirus Prevention in Kidney Transplant Recipients: A Single-Center Experience
}

\author{
Bhuwania $\mathbf{P}^{*}$ \\ Department of Nephrology, KG Hospital, Government \\ Arts College Road, Coimbatore, India \\ *Corresponding author: Puneet Bhuwania, \\ Department of Nephrology, KG Hospital, Government \\ Arts College Road, Coimbatore-641018, India
}

Received: October 11, 2021; Accepted: November 03, 2021; Published: November 10, 2021

\begin{abstract}
Background: Moderate-risk for Cytomegalovirus (CMV) infection includes patients with donor positive/recipient positive $(D+/ R+)$ or donor negative/ recipient positive antibody status $(\mathrm{D}-/ \mathrm{R}+)$. Guidelines recommend high-dose daily Valganciclovir (VGCV) as prophylaxis, which may be due to the paucity of data on the efficacy of every-other-day VGCV.
\end{abstract}

Methods: Our experience of using every-other-day VGCV as a prophylactic strategy in moderate risk Kidney Transplant Recipients (KTR) has been described. We retrospectively reviewed 86 moderate-risk KTR in our institution between 2018 and 2020. CMV infection at 6 months post-transplant was the primary endpoint. Graft survival, biopsy-proven rejection, opportunistic infections, Haematological adverse events, and mortality were also evaluated.

Results: CMV infection occurrence at 6 months was zero in our cohort Incidence of leukopenia was $13 \%$, BPAR- $31 \%$, OI- $33 \%$, and mortality being $3.5 \%$.

Conclusion: Every-Other-Day VGCV dosing can be an effective alternative in moderate risk KTR for CMV prevention.

Keywords: CMV prophylaxis; Valganciclovir; Kidney transplant recipients

\section{Abbreviations}

ATG-F: Anti-thymocyte globulin-Fresenius; BPAR: Biopsy Proven Acute Rejection; CD3: Cluster of Differentiation-3; CMV: Cytomegalovirus; D+/R-: Donor Positive/Recipient Negative; D-/ $\mathrm{R}+$ : Donor Negative/Recipient Positive; D+/R+: Donor Positive/ Recipient Positive; eGFR: Estimated Glomerular Filtration Rate; ug: Microgram; mg.h/L: Milligram.hours per Litre; MMF: Mycophenolate Mofetil; MPA AUC: Mycophenolic Acid Area under the Curve; ng/mL: Nanograms per millilitres; OI: Opportunistic Infection; PCR: Polymerase Chain Reaction; r-ATG: Rabbit Anti Thymocyte Globulin; TAC: Tacrolimus; VGCV: Valganciclovir; VZV: Varicellazoster

\section{Introduction}

Cytomegalovirus (CMV) is a well-established opportunistic infection confronted in the post-transplant setting. Its impact on graft survival, morbidity, and mortality in renal transplant recipients has always remained very significant $[1,2]$. Anti-CMV Immunoglobulin $\mathrm{G}$ (IgG) is detected in plasma in renal donors with prior CMV exposure when being evaluated for transplantation and so it is one of the multiple factors that can influence CMV emergence, others being CMV serological status of the recipient, the usage of pre-emptive therapy or prophylaxis and immune-suppressants [3]. None of the given guidelines accepts the prescription of Every-Other-Day dose of VGCV for Cytomegalovirus (CMV) prophylaxis, which is partly due to the lack of published data [1]. However, in a survey conducted internationally, nearly $30-40 \%$ of centers have reported using the low-dose regimen of $450 \mathrm{mg} /$ day in the moderate-risk population [4]. The safety and efficacy of low-dose VGCV for CMV prophylaxis in 478 intermediate-risk Kidney Transplant Recipients (KTR) has been reported by Heldenbrand et al. [5], who found no significant difference in the 12-month incidence of CMV disease (3.4\% vs. 3.5\%), rejection $(11.2 \%$ vs. $10.3 \%)$ or graft loss $(6.7 \%$ vs. $5.0 \%)$ between patients receiving high $v$ s. low-dose VGCV prophylaxis respectively. Khan et al. [6], also described a low incidence of CMV in $316 \mathrm{KTR}$ i.e. $2.7 \%$ in $\mathrm{D}+/ \mathrm{R}+, 4 \%$ in the $\mathrm{D}-/ \mathrm{R}+$ and $3 \%$ in $\mathrm{R}+$, where they used $450 \mathrm{mg} /$ day (adjusted to renal functions) of VGCV as prophylaxis. We used even lower doses of VGCV prophylaxis (defined as $450 \mathrm{mg}$ on Every-Other-Day with renal dose adjustment if needed) in moderate risk KTR at our institution and have presented our findings.

\section{Methods}

A retrospective analysis utilizing our institutional electronic medical record system of a cohort of $86 \mathrm{CMV} \mathrm{D+/R+KTR} \mathrm{between}$ 2018 and 2020 was done. The analysis was conducted following the Declaration of Helsinki, the International Conference on Harmonization, and Good Clinical Practice guidelines. Recipients included were live (related/directed - near and not near) or deceased complement-dependent cytotoxicity cross-match negative, HLA match or mismatch patients, along with $\mathrm{ABO}$-incompatible recipients, who underwent desensitization. All transplant recipients received a single dose of Anti-thymocyte globulin - Fresenius (ATG-F) IV (2-5 $\mathrm{mg} / \mathrm{kg}$ ) as a part of induction therapy along with methylprednisolone $500 \mathrm{mg}$ IV on postoperative days $0,1,2$ days as per unit protocol. Maintenance Immunosuppression included a combination of Anti- 
Table 1: Demographic and baseline characteristics.

\begin{tabular}{|c|c|}
\hline & $\begin{array}{c}\text { Alternative } \\
\mathrm{N}(\%)\end{array}$ \\
\hline Number & 86 \\
\hline Age $(\text { Years })^{\dagger}$ & $39.3 \pm 12.20$ \\
\hline Sex: Male & $69(80.0)$ \\
\hline Weight $(\mathrm{kg})^{\dagger}$ & $60.76 \pm 13.53$ \\
\hline \multicolumn{2}{|l|}{ Allograft Type } \\
\hline Live Donor & $71(82.5)$ \\
\hline Deceased Donor & $15(17.5)$ \\
\hline \multicolumn{2}{|l|}{ Live Donor } \\
\hline$A B O$ - incompatible & $8(9.3)$ \\
\hline ABO - compatible & $78(90.7)$ \\
\hline $\mathrm{HLA}$ Mismatch ${ }^{\dagger}$ & $6.26 \pm 2.37$ \\
\hline \multicolumn{2}{|l|}{ CMV Serostatus } \\
\hline D+/R+ (live) & $71(82.5)$ \\
\hline $\mathrm{R}+$ & $15(17.5)$ \\
\hline Induction r-ATG/ATG-F $(\mathrm{mg} / \mathrm{kg})^{\dagger}$ & $3.44 \pm 0.75$ \\
\hline \multicolumn{2}{|l|}{ Immuno-suppression } \\
\hline TAC/MMF & $81(94.2)$ \\
\hline CSA/MMF & $5(5.8)$ \\
\hline CD3 Level (cells/ml) ${ }^{\dagger}$ & $224.5 \pm 161.6$ \\
\hline
\end{tabular}

${ }^{\dagger}$ Mean \pm Standard Deviation; TAC: Tacrolimus; MMF: Mycophenolate Mofetil; CSA: Cyclosporine A; r-ATG/ATG-F: rabbit Anti-thymocyte globulin/Antithymocyte globulin - Fresenius.

proliferative agent i.e. mycophenolate mofetil $(30 \mathrm{mg} / \mathrm{kg})$, Calcineurin inhibitor i.e. Cyclosporine $(3-5 \mathrm{mg} / \mathrm{kg})$ or Tacrolimus $(0.15 \mathrm{mg} / \mathrm{kg})$ and Prednisolone (initially initiated at $20 \mathrm{mg} /$ day then was tapered to $2.5-5 \mathrm{mg} / \mathrm{d}$ at around 4 weeks post-transplant and remained the same during the study period.

The recipients received VGCV (defined as $450 \mathrm{mg}$ on every other day adjusted for renal function if needed) for CMV prophylaxis. Following transplantation, prophylaxis was given for the first 90100 days. The primary endpoint being CMV infection incidence till 6 months ( $\max$ incidence of CMV during this period) posttransplant defined as (i) Positive CMV Deoxyribonucleic acid (DNA) determination by Polymerase Chain Reaction (PCR) - which was performed based on clinical suspicion of the treating doctor (ii) Evidence of $\mathrm{CMV}$ with positive immunohistochemistry staining or viral inclusions on histology. The secondary endpoints included leucopenia episodes during the period of prophylaxis, graft survival, Biopsy-Proven Acute Rejection (BPAR), Opportunistic Infection (OI), and mortality.

\section{Results}

A total of 86 of moderate risk KTR were a part of our analysis. $80 \%$ of the patients were male and their mean age was 39 years (Table 1). All received VGCV prophylaxis for $90-100$ days.

\section{Efficacy}

CMV infection: The incidence of CMV infection at 6 months after transplant was zero in both live and deceased KTR - moderate risk (Table 2).
Table 2: Primary and Secondary endpoints.

\begin{tabular}{|c|c|}
\hline & $\begin{array}{c}\text { Alternative } \\
\mathrm{N}(\%)\end{array}$ \\
\hline CMV-PCR (Tested) at $6^{\text {th }}$ Month & 7 \\
\hline Positive & 0 \\
\hline \multicolumn{2}{|l|}{ Complications at $6^{\text {th }}$ Month } \\
\hline Diarrhea & $2(2.3)$ \\
\hline Fever & $16(18.6)$ \\
\hline Leukopenia & $11(12.8)$ \\
\hline \multicolumn{2}{|l|}{ Opportunistic Infection } \\
\hline Present & $24(32.5)$ \\
\hline Absent & $52(67.5)$ \\
\hline \multicolumn{2}{|l|}{ Mortality } \\
\hline Month 1 & $1(1.1)$ \\
\hline Month 3 & $1(1.1)$ \\
\hline Month 6 & $1(1.2)$ \\
\hline \multicolumn{2}{|l|}{ Serum Creatinine $(\mathrm{mg} / \mathrm{dl})^{\dagger}$} \\
\hline Month 1 & $1.32 \pm 0.86$ \\
\hline Month 3 & $1.29 \pm 0.41$ \\
\hline Month 6 & $1.22 \pm 0.39$ \\
\hline \multicolumn{2}{|l|}{ Biopsy Proven Graft Dysfunction } \\
\hline ATN & $9(10.0 \%)$ \\
\hline \multicolumn{2}{|l|}{ Rejection } \\
\hline ACR & $15(17.5 \%)$ \\
\hline AMR & $12(13.9 \%)$ \\
\hline Nil & $59(68.6 \%)$ \\
\hline
\end{tabular}

${ }^{\dagger}$ Mean \pm Standard Deviation; ATN: Acute Tubular Necrosis; ACR: Acute Cellular Rejection; AMR: Acute Antibody Mediated Rejection; CMV-PCR: Cytomegalovirus Polymerase Chain Reaction.

Safety and graft outcomes: The incidence of leukopenia was $13 \%$, BPAR - $31 \%$, OI - $33 \%$, and mortality is $3.5 \%$ (Table 2). None of the biopsies showed immunohistochemistry staining, viral inclusions, or any other features suggestive of CMV.

Others: With regards to immunosuppression, r-ATG/ATG-F was used as induction $(3.44 \pm 0.75 \mathrm{mg} / \mathrm{kg})$. These patients received a baseline steroid dose of $3.29 \pm 1.34 \mathrm{mg} / \mathrm{kg}$ with a tacrolimus trough levels $(10.51 \pm 2.48 \mathrm{ng} / \mathrm{ml}, 10.86 \pm 2.01 \mathrm{ng} / \mathrm{ml}$ and $10.21 \pm 1.76 \mathrm{ng} / \mathrm{ml}$ in $1^{\text {st }}, 2^{\text {nd }}$, and $3^{\text {rd }}$ months respectively), Cyclosporine levels (C0$176.8 \pm 39.78 \mathrm{ng} / \mathrm{ml}$ and $\mathrm{C} 0-157 \pm 30.75 \mathrm{ng} / \mathrm{ml}$ in the $1^{\text {st }}$ and $3^{\text {rd }}$ months respectively) and mycophenolic acid levels of $31.30 \pm 10.04 \mathrm{mg} . \mathrm{h} / \mathrm{l}$ during the prophylaxis (Table 3).

\section{Discussion}

Our analysis adds to the growing evidence supporting the effectiveness of using low-dose VGCV in preventing CMV infection in moderate-risk KTR. Zero cases of CMV 6 months posttransplantation were seen in our cohort. Furthermore, the incidence of leukopenia also remained small. As stated previously, little evidence favoring very low-dose VGCV for prophylaxis is available but even after that none of the studies have used such low doses of 450mg on Every-Other-Day. Many Pharmacokinetic (PK) studies have demonstrated that GCV exposure levels at oral GCV 3g/day 
Table 3: Characteristic of Immunosuppressive therapy in Patients during Followup.

\begin{tabular}{|c|c|}
\hline & Alternative \\
\hline \multicolumn{2}{|l|}{ Steroid dose } \\
\hline Prednisolone $(\mathrm{mg})^{\dagger}$ & $3.29 \pm 1.34$ \\
\hline \multicolumn{2}{|c|}{ Mean TAC trough conc. (ng/ml) ${ }^{\dagger}$} \\
\hline Month 1 & $10.51 \pm 2.48$ \\
\hline Month 2 & $10.86 \pm 2.01$ \\
\hline Month 3 & $10.21 \pm 1.76$ \\
\hline \multicolumn{2}{|c|}{ Mean CSA trough conc. $(\mathrm{ng} / \mathrm{ml})^{\dagger}$} \\
\hline Month 1 & $176.8 \pm 39.78$ \\
\hline Month 3 & $157 \pm 30.75$ \\
\hline MPA AUC (mg.h/l $)^{\dagger}$ & $31.30 \pm 10.04$ \\
\hline
\end{tabular}

${ }^{\dagger}$ Mean \pm Standard Deviation; TAC: Tacrolimus; CSA: Cyclosporine A; MPA-AUC: Mycophenolate Acid Area Under Curve.

dose can be achieved by low-dose VGCV. Our analyses inferred that low-dose VGCV can provide enough drug levels for effective CMV prophylaxis [7-9]. Kalil et al. demonstrated in a meta-analysis, that equivalent efficacy for CMV prophylaxis can be provided by either of the low or high-dose VGCV regimens (97\% statistical power) [10]. With growing clinical evidence, which is supported by the PK data for the use of low-dose VGCV, the Moderate risk population can be reasonably evaluated by this regimen of Every-Other-Day dose.

\section{Limitations}

This is a single-center experience, hence may not apply to other transplant units. Data were retrospectively collected, hence did not have a group receiving the standard doses (as per guidelines) of VGCV as a comparison. In patients whose renal functions fluctuate, especially KTR, renal dosing of medication is a highly specific task that requires the use of elements beyond just serum creatinine and eGFR, these adjustments were made at the discretion of the treating doctor and hence not controlled. Finally, As CMV events are rare in our transplant population and testing was done based on clinical judgment; it limited our ability to get precise results. In summary, a low incidence of CMV infection, less adverse drug effects, and expected cost savings support the use of Every-Other-Day dosing of VGCV as CMV prophylaxis in moderate-risk KTR. Although a formal pharmaco-economic evaluation was never completed in our analysis, an undeniable superiority of the Every-Other-Day VGCV regimen was its cost advantage. Even though the cohort included patients of a moderate-risk group, all of them received ATG as induction therapy and were maintained on high CNI trough levels during the duration of valganciclovir prophylaxis in the post-transplant period hence they had a higher risk of developing CMV infection than the standard moderate risk group patients. In our opinion, such very lowdose VGCV prophylaxis in D+/R+ KTR can be considered as a viable option in future guidelines.

\section{References}

1. Kotton CN. CMV: Prevention, diagnosis and therapy. Am J Transplant. 2013; 13: $24-40$.

2. Fehr $\mathrm{T}$, Cippà PE, Mueller NJ. Cytomegalovirus post kidney transplantation: prophylaxis versus pre-emptive therapy? Transplant International. 2015; 28 : 1351-1356.

3. L Parreira, M Bruges, A Gaspar, A Weigert, D Machado. Prevention of cytomegalovirus disease in renal transplantation: single-center experience. Transplant. 2009; 41: 877-879.

4. Le Page AK, Jager MM, Kotton CN, Simoons-Smit A, Rawlinson WD International survey of cytomegalovirus management in solid organ transplantation after the publication of consensus guidelines. Transplantation. 2013; 95: 1455-1460.

5. Heldenbrand S, Li C, Cross RP, et al. Multicenter evaluation of efficacy and safety of low-dose versus high-dose valganciclovir for prevention of CMV disease in donor and recipient positive $(\mathrm{D}+/ \mathrm{R}+)$ renal transplant recipients. Transpl Infect Dis. 2016; 18: 904-912.

6. Khan S, Fischman C, Huprikar S. Low-dose valganciclovir prophylaxis for cytomegalovirus in intermediate-risk $(\mathrm{R}+)$ renal transplant recipients: Singlecenter experience. Transplant Infectious Disease. 2017; 19: e12780.

7. Boeckh M, Gooley TA, Myerson D, Cunningham T, Schoch G, Bowden RA. Cytomegalovirus pp65 antigenemia-guided early treatment with ganciclovir versus ganciclovir at engraftment after allogeneic marrow transplantation: a randomized double-blind study. Blood. 1996; 88: 4063-4071.

8. Pescovitz MD, Rabkin J, Merion RM, et al. Valganciclovir results in improved oral absorption of ganciclovir in liver transplant recipients. Antimicrob Agents Chemother. 2000; 44: 2811-2815.

9. Chamberlain CE, Penzak SR, Alfaro RM, et al. Pharmacokinetics of low and maintenance dose valganciclovir in kidney transplant recipients. Am J Transplant. 2008; 8: 1297-1302.

10. Kalil AC, Levitsky J, Lyden E, Stoner J, Freifeld AG. Meta-analysis: the efficacy of strategies to prevent organ disease by cytomegalovirus in solid organ transplant recipients. Ann Intern Med. 2005; 143: 870-880. 